MATHEMATICS OF COMPUTATION

Volume 71, Number 238 , Pages 669-681

S 0025-5718(01)01383-7

Article electronically published on November 28, 2001

\title{
INVERSE AND SATURATION THEOREMS FOR RADIAL BASIS FUNCTION INTERPOLATION
}

\author{
ROBERT SCHABACK AND HOLGER WENDLAND
}

\begin{abstract}
While direct theorems for interpolation with radial basis functions are intensively investigated, little is known about inverse theorems so far. This paper deals with both inverse and saturation theorems. For an inverse theorem we especially show that a function that can be approximated sufficiently fast must belong to the native space of the basis function in use. In case of thin plate spline interpolation we also give certain saturation theorems.
\end{abstract}

\section{INTRODUCTION}

Direct and inverse theorems play an important role in classical approximation theory. Examples can be found in [2, 10]. The main idea can be described as follows. Suppose the elements of a linear space $(V,\|\cdot\|)$ should be approximated by elements of finite dimensional subspaces $V_{h} \subseteq V$, where $h$ serves as a discretization index. Denote the approximation process by $S_{h}: V \rightarrow V_{h}$. Then the direct theorems conclude error estimates from additional information on the elements to be approximated: If $f$ is an element of a subspace $\mathcal{G} \subseteq V$, then the error can be bounded by

$$
\left\|f-S_{h} f\right\| \leq c_{f} h^{\mu} .
$$

On the other hand the inverse theorems try to conclude information on $f$ from the way $f$ can be approximated: If $f \in V$ satisfies (1.1), then $f$ must belong to a certain subspace $\mathcal{G} \subseteq V$. The situation is optimal if the subspaces and the approximation orders coincide in both the direct and the inverse theorems. The subspace $\mathcal{G}$ consists normally of smooth functions.

Finally, saturation theorems give upper bounds on the possible approximation order: If $f \in \mathcal{G}$ can be approximated by

$$
\left\|f-S_{h} f\right\| \leq c_{f} h^{\nu}
$$

where $\nu$ is a certain number larger than $\mu$, then $f$ must belong to a trivial subspace $\mathcal{N} \subseteq V$.

It is the aim of this paper to give both inverse and saturation theorems in the context of radial basis function interpolation. In case of direct and inverse theorems we shall take the native space $\mathcal{G}_{\Omega, \Phi}$, which we introduce in the third section, as the space $\mathcal{G}$ of smoother functions. We will look for the approximation order we can

Received by the editor February 10, 2000.

2000 Mathematics Subject Classification. Primary 41A05, 41A17, 41A27, 41A30, 41A40.

Key words and phrases. Positive definite functions, approximation orders.

(C)2001 American Mathematical Society 
achieve from this fact and for the order we need to show that a function belongs to the native space.

Our main results in case of inverse theorems deal with basis functions that generate Sobolev spaces as their native spaces. Thus the native space is indeed a certain smoothness class. But even in the case of Gaussians where the native space is rather small, it contains at least all functions with a compactly supported Fourier transform. Thus the native space for Gaussians contains at least the class of functions for which Shannon's sampling theorem holds.

In case of saturation theorems we restrict ourselves to thin plate spline interpolation and show that functions that can be approximated with a high order are necessarily polyharmonic functions.

We also provide another kind of inverse theorem. Our corresponding result does not concern the functions to be approximated, but the basis function itself. It allows us to draw conclusions on the smoothness of the basis function from estimates on the power function.

Finally, we provide a new characterization of the native space generated by radial basis function interpolants. This gives us a numerical tool to test whether an unknown function belongs to the native space (and thus in several cases to a Sobolev space) or not.

\section{RADIAL BASIS FUNCTION APPROXIMATION}

The theory of interpolation by radial basis functions has become popular in recent years for reconstructing multivariate functions from scattered data. The starting point of the reconstruction process is the choice of a conditionally positive definite function $\Phi: \mathbb{R}^{d} \rightarrow \mathbb{R}$.

Definition 2.1. A continuous and even function $\Phi: \mathbb{R}^{d} \rightarrow \mathbb{R}$ is said to be conditionally positive definite of order $m \in \mathbb{N}_{0}$, iff for all $N \in \mathbb{N}$, for all sets of pairwise distinct centers $X=\left\{x_{1}, \ldots, x_{N}\right\} \subseteq \mathbb{R}^{d}$, and for all $\alpha \in \mathbb{R}^{N} \backslash\{0\}$ satisfying

$$
\sum_{j=1}^{N} \alpha_{j} p\left(x_{j}\right)=0 \quad \text { for all } p \in \mathcal{P}_{m}^{d},
$$

the quadratic form

$$
\sum_{j, k=1}^{N} \alpha_{j} \alpha_{k} \Phi\left(x_{j}-x_{k}\right)
$$

is positive. Here, $\mathcal{P}_{m}^{d}$ denotes the set of all $d$-variate polynomials with a total degree less then $m$. We will denote the set of all conditionally positive definite functions of order $m$ by $\operatorname{cpd}(m)$. A conditionally positive definite function of order $m=0$ is also called a positive definite function.

Having a $\Phi \in \operatorname{cpd}(m)$, the interpolant $s_{f, X}$ to a function $f$ in $X=\left\{x_{1}, \ldots, x_{N}\right\}$ is given by

$$
s_{f, X}(x)=\sum_{j=1}^{N} \alpha_{j} \Phi\left(x-x_{j}\right)+\sum_{j=1}^{Q} \beta_{j} p_{j}(x),
$$


where $p_{1}, \ldots, p_{Q}$ form a basis of $\mathcal{P}_{m}^{d}$. To cope with the additional degrees of freedom, the interpolation conditions

$$
s_{f, X}\left(x_{j}\right)=f\left(x_{j}\right), \quad 1 \leq j \leq N,
$$

are completed by the further conditions

$$
\sum_{j=1}^{N} \alpha_{j} p_{k}\left(x_{j}\right)=0, \quad 1 \leq k \leq Q .
$$

In this paper we are mainly interested in positive definite functions that possess a Fourier transform $\widehat{\Phi}$ that decays only algebraically, i.e., there exist constants $0<c_{1} \leq c_{2}$ with

$$
c_{1}(1+\|\omega\|)_{2}^{-d-s_{\infty}} \leq \widehat{\Phi}(\omega) \leq c_{2}(1+\|\omega\|)_{2}^{-d-s_{\infty}}
$$

for $\|\omega\|_{2} \rightarrow \infty$. The upper bound is important for the direct theorems, while the lower bound is necessary for the inverse theorems. This decay condition is, for instance, covered by Sobolev splines and compactly supported radial basis functions of minimal degree (cf. [16]). In this situation the definition of the native space via Fourier transform shows immediately that the global native space $\mathcal{G}_{\mathbb{R}^{d}}$, coincides with the classical global Sobolev space $\mathcal{G}_{\mathbb{R}^{d}, \Phi}=W_{2}^{\left(d+s_{\infty}\right) / 2}\left(\mathbb{R}^{d}\right)$. A more abstract definition of native spaces and certain extension arguments also allow us to give direct and inverse theorems for local native spaces which coincide with local Sobolev spaces $W_{2}^{\left(d+s_{\infty}\right) / 2}(\Omega)$ under very mild conditions on the domain $\Omega$.

But our methods do not work only for functions generating Sobolev spaces, they work also for other basis functions. Thus we shall state the inverse theorems also in the case of exponentially decaying Fourier transforms, which covers Gaussians and inverse multiquadrics.

\section{Direct THEOREMS}

There are several papers dealing with direct theorems, but only a few have tried to establish inverse theorems. We will briefly repeat direct theorems as far as we need them for our further analysis.

To state error estimates two preliminary steps have to be done. On the one hand the function space has to be introduced for which the error bounds shall apply. On the other hand a measure of the data density has to be given. We start with the function space. Almost every paper (cf. [7, 8, 9, 12, 16, 17]) that deals with direct theorems for scattered data interpolation by radial basis functions uses the native space as the space of smooth functions. Even in the case of "radial" functions on the sphere the concept of native spaces is carried over $([5,4])$. The easiest way to introduce native spaces on $\mathbb{R}^{d}$ is by a Fourier transform. Suppose $\Phi$ is a positive definite function with a nonnegative, nonvanishing Fourier transform $\widehat{\Phi}$. Then the native space $\mathcal{G}_{\mathbb{R}^{d}, \Phi}$ consists of all functions $f: \mathbb{R}^{d} \rightarrow \mathbb{R}$ which can be recovered via

$$
f(x)=(2 \pi)^{-d} \int_{\mathbb{R}^{d}} \widehat{f}(\omega) e^{i x^{T} \omega} d \omega,
$$

where $\widehat{f}$ satisfies

$$
\frac{\widehat{f}}{\sqrt{\widehat{\Phi}}} \in L_{2}\left(\mathbb{R}^{d}\right) .
$$


It follows immediately from this that the native space is a space of smooth functions, since in general the Fourier transform of the basis function decreases and thus $\widehat{f}$ has to decrease as well. Furthermore, it is obvious that if $\widehat{\Phi}$ satisfies (2.4), the native space coincides with a classical Sobolev space.

Another way to introduce native spaces which also takes a local domain $\Omega$ into account is the following one. For the equivalence of both definitions see $8,9,13,14$

Let $\Omega \subseteq \mathbb{R}^{d}$ be given. Let us denote by

$$
\left(\mathcal{P}_{m}^{d}\right)_{\Omega}^{\perp}=\left\{\lambda_{\alpha, X}=\sum_{j=1}^{M} \alpha_{j} \delta_{x_{j}}: M \in \mathbb{N}, \alpha_{j} \in \mathbb{R}, x_{j} \in \Omega, \lambda_{\alpha, X} \mid \mathcal{P}_{m}^{d} \equiv 0\right\}
$$

the set of all point evaluation functionals of finite support in $\Omega$ vanishing on $\mathcal{P}_{m}^{d}$. Every conditionally positive definite function $\Phi$ of order $m$ allows us to equip $\left(\mathcal{P}_{m}^{d}\right)_{\Omega}^{\perp}$ with an inner product

$$
(\lambda, \mu)_{\Phi}=\lambda^{x} \mu^{y} \Phi(x-y),
$$

where $\lambda^{x}$ means the action of $\lambda$ with respect to the variable $x$.

Then we can introduce the function space

$$
\mathcal{G}_{\Omega, \Phi}=\left\{f \in C(\Omega):|\lambda(f)| \leq c_{f}\|\lambda\|_{\Phi} \text { for all } \lambda \in\left(\mathcal{P}_{m}^{d}\right)_{\Omega}^{\perp}\right\}
$$

We denote the smallest constant $c_{f}$ in the definition of $\mathcal{G}_{\Omega, \Phi}$ by $\|f\|_{\Phi}$, i.e.,

$$
\|f\|_{\Phi}:=\max _{\lambda \in\left(\mathcal{P}_{m}^{d}\right) \frac{\perp}{\Omega} \backslash\{0\}} \frac{|\lambda(f)|}{\|\lambda\|_{\Phi}} .
$$

Then $\|\cdot\|_{\Phi}$ is a semi-norm on $\mathcal{G}_{\Omega, \Phi}$ with null space $\mathcal{P}_{m}^{d}$. Thus

$$
\mathcal{F}_{\Omega, \Phi}:=\mathcal{G}_{\Omega, \Phi} / \mathcal{P}_{m}^{d}
$$

is a normed linear space which turns out to be complete.

Not only is the space $\mathcal{P}_{m}^{d}$ a subspace of $\mathcal{G}_{\Omega, \Phi}$, but also all interpolating functions (2.1) are contained in it.

Lemma 3.1. The map

$$
\begin{aligned}
F:\left(\mathcal{P}_{m}^{d}\right)_{\Omega}^{\perp} & \rightarrow F\left(\left(\mathcal{P}_{m}^{d}\right)_{\Omega}^{\perp}\right) \subseteq G_{\Omega, \Phi} \\
\lambda_{\alpha, X} & \mapsto \lambda_{\alpha, X}^{y} \Phi(\cdot-y)
\end{aligned}
$$

is well defined and bijective. Furthermore, we have the relations

$$
\left\|\lambda_{\alpha, X}\right\|_{\Phi}=\left\|F\left(\lambda_{\alpha, X}\right)\right\|_{\Phi}
$$

and

$$
\lambda_{\alpha, X}\left(F\left(\lambda_{\beta, Y}\right)\right)=\left(\lambda_{\alpha, X}, \lambda_{\beta, Y}\right)_{\Phi}=\lambda_{\beta, Y}\left(F\left(\lambda_{\alpha, X}\right)\right) .
$$

The proof is straightforward and will be omitted.

The first step in bounding the interpolation error is to define the power function as the norm of the pointwise error functional

$$
P_{X, \Phi}(x)=\sup _{f \in \mathcal{G}_{\Omega, \Phi} \backslash \mathcal{P}_{m}^{d}} \frac{\left|f(x)-s_{f, X}(x)\right|}{\|f\|_{\Phi}},
$$

which leads immediately to

$$
\left|f(x)-s_{f, X}(x)\right| \leq P_{X, \Phi}(x)\|f\|_{\Phi}
$$


Then the power function has to be bounded in terms of the fill distance, defined by

$$
h_{X, \Omega} \equiv h_{X}:=\sup _{x \in \Omega} \min _{x_{j} \in X}\left\|x-x_{j}\right\|_{2},
$$

which was done in [17, for instance.

Theorem 3.2. Let $\Phi \in \operatorname{cpd}(m)$ satisfy (2.4). Let $\Omega$ be a bounded and open domain satisfying an interior cone condition. Then there exist constants $h_{0}, C$, such that for all sets of centers $X$ with $h_{X} \leq h_{0}$ and all $x \in \Omega$ the power function can be bounded by

$$
P_{X, \Phi}(x) \leq C h_{X}^{s_{\infty} / 2}
$$

yielding the error bound

$$
\left\|f-s_{f, X}\right\|_{L_{\infty}(\Omega)} \leq C h_{X}^{s_{\infty} / 2}\|f\|_{\Phi}
$$

for $f \in \mathcal{G}_{\Omega, \Phi}$.

Actually, in 17. the theorem is stated in a more localized version, but the proof holds true in this situation. There are several other papers giving error bounds of this form; some of them are 10 3] 7 , 15.

Next, we need a stability result on the interpolation process. Therefore, we define the separation distance

$$
q_{X}:=\frac{1}{2} \min _{j \neq k}\left\|x_{j}-x_{k}\right\|_{2}
$$

and cite from 11

Theorem 3.3. Let $\Phi \in \operatorname{cpd}(m)$ satisfy the decay condition (2.4). For the set of centers $X=\left\{x_{1}, \ldots, x_{N}\right\} \subseteq \Omega$ denote by $A_{X, \Phi}$ the matrix

$$
A_{X, \Phi}=\left(\Phi\left(x_{j}-x_{k}\right)\right)_{1 \leq j, k \leq N}
$$

and by $\gamma_{X}$ the smallest constant with $\alpha^{T} A_{X, \Phi} \alpha \geq \gamma_{X}\|\alpha\|_{2}^{2}$ for $\alpha$ satisfying (2.3). Then the following holds true:

1) (Stability) For all $\alpha \in \mathbb{R}^{N}$ satisfying (2.3) we have

$$
\alpha^{T} A_{X, \Phi} \alpha \geq \gamma_{X}\|\alpha\|_{2}^{2} \geq c_{\Phi} q_{X}^{s_{\infty}}\|\alpha\|_{2}^{2}
$$

and therefore

$$
\left\|\left(A_{X, \Phi} \mid V_{X}\right)^{-1}\right\|_{2,2} \leq c_{\Phi} q_{X}^{-s_{\infty}}
$$

with $V_{X}:=\left\{\alpha: \lambda_{\alpha, X} \in\left(\mathcal{P}_{m}^{d}\right)_{\Omega}^{\perp}\right\}$ and a constant $c_{\Phi}$ depending only on $\Phi$.

2) (Uncertainty relation) For all $x \in \Omega \backslash X$ we have

$$
P_{X, \Phi}^{2}(x) \geq \gamma_{X \cup\{x\}}
$$

\section{INVERSE THEOREMS CONCERNING $\Phi$}

Principally, there are two possibilities for stating inverse theorems for interpolation by radial basis functions. The first one is based only on the basis function $\Phi$ and draws conclusions on the basis function from the fact that the power function can be bounded like (3.1). This will be done in this section. The second is to draw conclusions on $f$ from estimates like (3.2) which will be the subject of the sixth section. 
Theorem 4.1. Let $\Phi \in \operatorname{cpd}(m)$ satisfy (2.4). Let $\Omega$ be bounded and open, satisfying an interior cone condition. If there exist constants $\beta, c>0$ such that the power function $P_{X, \Phi}$ can be bounded by

$$
\left\|P_{X, \Phi}\right\|_{L_{\infty}(\Omega)} \leq c h_{X}^{\beta / 2}
$$

for all sets $X \subseteq \Omega$ with sufficiently small $h_{X}$, then

$$
s_{\infty} \geq \beta
$$

must be satisfied.

Proof. On account of the conditions on $\Omega$ there exists a $\delta>0$ and quasi-uniform sets $X=\left\{x_{1}, \ldots, x_{N}\right\} \subseteq \Omega$ with respect to this $\delta>0$. Here, we call a set of pairwise distinct centers $X=\left\{x_{1}, \ldots, x_{N}\right\} \subseteq \Omega$ quasi-uniform with respect to $\delta>0$, iff

1) $X \backslash\left\{x_{j}\right\}$ is $\mathcal{P}_{m}^{d}$-unisolvent for $1 \leq j \leq N$, i.e., zero is the only polynomial from $\mathcal{P}_{m}^{d}$ that vanishes on $X \backslash\left\{x_{j}\right\}$,

2) $q_{X} \geq \delta h_{X}$.

Then we have (cf. [11]) $h_{X \backslash\left\{x_{j}\right\}} \leq 2 h_{X}$ for $h_{X}$ sufficiently small. Therefore we can use (2.4), (4.1) and the Uncertainty Relation (3.3) to derive

$$
c 2^{\beta} h_{X}^{\beta} \geq c h_{X \backslash\left\{x_{j}\right\}}^{\beta} \geq P_{X \backslash\left\{x_{j}\right\}}^{2}\left(x_{j}\right) \geq \gamma_{X} \geq c_{\Phi} q_{X}^{s_{\infty}} \geq c_{\Phi} \delta^{s_{\infty}} h_{X}^{s_{\infty}} .
$$

Choosing a sequence of such $X$ where $h_{X} \rightarrow 0$ leads to $\beta \leq s_{\infty}$.

Theorem 4.1 shows that the decay (4.1) of the power function determines the decay of the generalized Fourier transform of the basis function and therefore the smoothness of the basis function itself. It also shows that there is no possibility to improve error estimates of the form (3.2) based on upper bounds of the power function.

\section{Characterization of the native Space}

Our next result characterizes the functions $f$ from the native space $\mathcal{G}_{\Omega, \Phi}$ by uniform boundedness of their interpolating functions with respect to the semi-norm of the native space.

Theorem 5.1. Denote by $s_{f, X}$ the interpolant (2.1) to a function $f \in C(\Omega)$ on $X$ using a basis function $\Phi \in \operatorname{cpd}(m)$. Then $f$ belongs to the native space $\mathcal{G}_{\Omega, \Phi}$ if and only if there exists a constant $c_{f}$ such that $\left\|s_{f, X}\right\|_{\Phi} \leq c_{f}$ for all $X \subseteq \Omega$.

Proof. Assume $f \in \mathcal{G}_{\Omega, \Phi}$. Then $s_{f, X}$ is the best approximation to $f$ from $\operatorname{span}\{\Phi(\cdot-x): x \in X\}+\mathcal{P}_{m}^{d}$ with respect to the $\|\cdot\|_{\Phi^{-} \text {-semi-norm. Thus we }}$ have

$$
\left\|f-s_{f, X}\right\|_{\Phi}^{2}+\left\|s_{f, X}\right\|_{\Phi}^{2}=\|f\|_{\Phi}^{2},
$$

which gives the bound $\left\|s_{f, X}\right\|_{\Phi} \leq\|f\|_{\Phi}$ at once. 
Now, let us assume $\left\|s_{f, X}\right\|_{\Phi} \leq c_{f}$ for all $X \subseteq \Omega$. For an arbitrary

$$
\lambda_{\alpha, X}:=\sum_{j=1}^{N} \alpha_{j} \delta_{x_{j}} \in\left(\mathcal{P}_{m}^{d}\right)_{\Omega}^{\perp},
$$

we choose $s_{f, X}$ to be the interpolant on $X$ to $f$ satisfying the interpolation conditions (2.2) and (2.3). Then $s_{f, X}$ belongs to $\mathcal{G}_{\Omega, \Phi}$ and we have

$$
\lambda_{\alpha, X}\left(f-s_{f, X}\right)=0 .
$$

Thus we can estimate

$$
\begin{aligned}
\left|\lambda_{\alpha, X}(f)\right| & \leq\left|\lambda_{\alpha, X}\left(f-s_{f, X}\right)\right|+\left|\lambda_{\alpha, X}\left(s_{f, X}\right)\right| \\
& \leq\left\|\lambda_{\alpha, X}\right\|_{\Phi}\left\|s_{f, X}\right\|_{\Phi} \\
& \leq c_{f}\left\|\lambda_{\alpha, X}\right\|_{\Phi} .
\end{aligned}
$$

As this holds for all $\lambda_{\alpha, X}$ we have $f \in \mathcal{G}_{\Omega, \Phi}$.

Note that Theorem 5.1 can be used as a numerical test to assess whether a function $f$ belongs to the native space. It is based on function values only.

\section{INVERSE THEOREMS CONCERNING $f$}

Now we draw conclusions about a function from $L_{\infty}$-convergence orders of its interpolants. To be more precise, we show that a function $f \in C(\Omega)$ which can be approximated sufficently fast by radial basis function interpolants in the $L_{\infty}$-norm must belong to the native space of the basis function.

Theorem 6.1. Let $\Omega \subseteq \mathbb{R}^{d}$ be a bounded and open domain satisfying an interior cone condition. The positive definite basis function $\Phi$ should satisfy the decay condition (2.4). Suppose further that for some $f \in C(\Omega)$ there exist constants $\mu>0$ and $c_{f}>0$ such that $\left\|f-s_{f, X}\right\|_{L_{\infty}(\Omega)} \leq c_{f} h_{X}^{\mu}$ for all $X \subseteq \Omega$ with $h_{X}$ sufficiently small. If $2 \mu>s_{\infty}+d$, then $f$ must belong to the native space $\mathcal{G}_{\Omega, \Phi}$.

Proof. All sets of centers $X$ that may appear in this proof shall be quasi-uniform with respect to a fixed $\delta>0$.

Every interpolant $s_{f, X}$ defines a linear functional $\lambda_{\alpha, X}=\sum_{j=1}^{N} \alpha_{j} \delta_{x_{j}}$. From Theorem 3.3 and Lemma 3.1 we have

$$
\begin{aligned}
\left\|s_{f, X}\right\|_{\Phi}^{2} & =\left\|\lambda_{\alpha, X}\right\|_{\Phi}^{2} \\
& =\alpha^{T} A_{X, \Phi} \alpha \\
& =\alpha^{T} A_{X, \Phi} A_{X, \Phi}^{-1} A_{X, \Phi} \alpha \\
& \leq\left\|A_{X, \Phi}^{-1}\right\|_{2,2}\left\|A_{X, \Phi} \alpha\right\|_{L_{2}(X)}^{2} \\
& =\left\|A_{X, \Phi}^{-1}\right\|_{2,2}\left\|s_{f, X}\right\|_{L_{2}(X)}^{2} .
\end{aligned}
$$

If $X \subseteq Y$ the difference $s_{f, Y}-s_{f, X}$ can be interpreted as the interpolating function on $Y$ to itself. This leads us to

$$
\begin{aligned}
\left\|s_{f, Y}-s_{f, X}\right\|_{\Phi}^{2} & \leq\left\|A_{Y, \Phi}^{-1}\right\|_{2,2}\left\|s_{f, Y}-s_{f, X}\right\|_{L_{2}(Y)}^{2} \\
& \leq \frac{1}{\gamma_{Y}} \sum_{y \in Y}\left|f(y)-s_{f, X}(y)\right|^{2} \\
& \leq c_{\Phi}^{-1} q_{Y}^{-s_{\infty}}|Y| c_{f}^{2} h_{X}^{2 \mu}
\end{aligned}
$$


In what follows $c$ will denote a generic constant. Now we consider a special family of quasi-uniform sets of centers. We assume $X_{n}$ to satisfy $\left|X_{n}\right| \leq c 2^{\text {nd }}$ and

$$
c_{1} 2^{-n} \leq q_{X_{n}} \leq h_{X_{n}} \leq c_{2} 2^{-n} .
$$

Such a choice is always possible because of the assumption made on $\Omega$. If we take $X=X_{k} \subseteq Y=X_{n}$ with $n \geq k$ we get

$$
\begin{aligned}
\left\|s_{f, X_{n}}-s_{f, X_{k}}\right\|_{\Phi}^{2} & \leq c 2^{s_{\infty} n+d n-2 \mu k} \\
& =c 2^{\left(d+s_{\infty}\right) n-2 \mu k} \\
& =c 2^{2 \mu(n-k)-2 \sigma n},
\end{aligned}
$$

where $\sigma>0$ is defined by $d+s_{\infty}+2 \sigma=2 \mu$. Thus we can estimate the $\Phi$-norm of two succeeding interpolants by

$$
\left\|s_{f, X_{k+1}}-s_{f, X_{k}}\right\|_{\Phi} \leq c 2^{-k \sigma} .
$$

A telescoping sum argument leads to

$$
\begin{aligned}
\left\|s_{f, X_{K}}\right\|_{\Phi} & \leq \sum_{k=0}^{K}\left\|s_{f, X_{k+1}}-s_{f, X_{k}}\right\|_{\Phi}+\left\|s_{f, X_{0}}\right\|_{\Phi} \\
& \leq c \sum_{j=0}^{\infty} 2^{-\sigma k}+\left\|s_{f, X_{0}}\right\|_{\Phi} . \\
& \leq \frac{c}{1-2^{-\sigma}}+\left\|s_{f, X_{0}}\right\|_{\Phi} .
\end{aligned}
$$

Thus, the sequence $\left\|s_{f, X_{k}}\right\|_{\Phi}$ is bounded. But for $n \geq k$ the interpolant $s_{f, X_{k}}$ is also the interpolant to $s_{f, X_{n}}$ and therefore a best approximant to $s_{f, X_{n}}$ from $S\left(X_{k}\right):=\operatorname{span}\left\{\Phi(\cdot-x): x \in X_{k}\right\}+\mathcal{P}_{m}^{d}$. This leads to

$$
\left\|s_{f, X_{n}}-s_{f, X_{k}}\right\|_{\Phi}^{2}+\left\|s_{f, X_{k}}\right\|_{\Phi}^{2}=\left\|s_{f, X_{n}}\right\|_{\Phi}^{2},
$$

which shows that the sequence $\left\|s_{f, X_{k}}\right\|_{\Phi}$ is also increasing and therefore convergent. Furthermore, (6.2) implies that $s_{f, X_{n}}$ is a Cauchy sequence in $\mathcal{G}_{\Omega, \Phi}$ with a limit $\tilde{s}$.

Finally, we have to show that $f$ coincides on $\Omega$ with $\tilde{s}$. Since we work with a positive definite function the point evaluation functional $\delta_{x}, x \in \Omega$, is continuous on the native space $\mathcal{G}_{\Omega, \Phi}$. This gives

$$
\begin{aligned}
\left|\delta_{x}(f-\tilde{s})\right| & \leq\left|\delta_{x}\left(f-s_{f, X_{n}}\right)\right|+\left|\delta_{x}\left(s_{f, X_{n}}-\tilde{s}\right)\right| \\
& \leq\left\|\delta_{x}\right\|_{\Phi}\left\|\tilde{s}-s_{f, X_{n}}\right\|_{\Phi}+\left|\left(f-s_{f, X_{n}}\right)(x)\right| \\
& \leq\left\|\delta_{x}\right\|_{\Phi}\left\|\tilde{s}-s_{f, X_{n}}\right\|_{\Phi}+c h_{X_{n}}^{\mu} .
\end{aligned}
$$

Thus we can derive $f(x)=\tilde{s}(x)$ for all $x \in \Omega$.

Note that there is a gap of $d / 2$ between the necessary and sufficient approximation order for functions in the native space $\mathcal{G}_{\Omega, \Phi}$. A closer look shows that the direct Theorem 3.2 implies for $f \in \mathcal{G}_{\Omega, \Phi}$ :

$$
\left\|f-s_{f, X}\right\|_{L_{\infty}(\Omega)} \leq C_{\Phi} h^{s_{\infty} / 2}\left\|f-s_{f, X}\right\|_{\Phi} .
$$

The $h^{s_{\infty} / 2}$ term comes from the estimate on the power function and is optimal in the sense of Theorem 4.1 On the other hand

$$
\left\|f-s_{f, X}\right\|_{L_{\infty}(\Omega)} \leq C_{f} h^{s_{\infty} / 2} h^{d / 2+\varepsilon}
$$


is so far necessary for showing $f \in \mathcal{G}_{\Omega, \Phi}$ via Theorem 6.1. Thus the gap could be closed either by showing

$$
\left\|f-s_{f, X}\right\|_{\Phi} \leq C_{f} h^{d / 2+\varepsilon},
$$

or by improving our inverse theorem.

Before we come to inverse theorems for Gaussian and inverse multiquadrics, let us remark that equation (6.1) can be rewritten as

$$
\left\|s_{f, X}\right\|_{\Phi} \leq c h_{X}^{-\left(s_{\infty}+d\right) / 2}\left\|s_{f, X}\right\|_{L_{\infty}(\Omega)}
$$

which can be seen as a kind of Bernstein inequality.

Now, let us assume for the rest of the section that the Fourier transform satisfies

$$
\widehat{\Phi}(\omega) \geq c e^{-\tilde{c}_{1}\|\omega\|_{2}^{\alpha}} .
$$

This leads to estimates of the form

$$
\left\|A_{X, \Phi}^{-1}\right\|_{2,2} \leq c e^{c_{1} h_{X}^{-\alpha}},
$$

where $c$ always denotes a generic constant. In case of multiquadrics and Gaussians the constants $\alpha, \tilde{c}_{1}$ and $c_{1}$ can be found in [11.

Theorem 6.2. Let $\Omega \subset \mathbb{R}^{d}$ be a bounded and open domain satisfying an interior cone condition. The positive definite basis function $\Phi$ should satisfy the decay condition (6.4). Suppose further that for some $f \in C(\Omega)$ there exist constants $c_{2}>c_{1}$ and $c_{f}>0$ such that $\left\|f-s_{f, X}\right\|_{L_{\infty}(\Omega)} \leq c_{f} e^{-c_{2} h_{X}^{\alpha}}$ for all $X \subseteq \Omega$ with $h_{X}$ sufficiently small. Then $f$ must belong to the native space $\mathcal{G}_{\Omega, \Phi}$.

Proof. The proof of Theorem 6.1 applies completely if we show that the native space norm of the difference of two interpolants can be bounded in such a way that the telescoping sum argument still works. But this is the case: for $X \subseteq Y$ we can derive

$$
\begin{aligned}
\left\|s_{f, X}-s_{f, Y}\right\|_{\Phi} & \leq c e^{c_{1} h_{X}^{-\alpha}} \sum_{y \in Y}\left|f(y)-s_{f, X}(y)\right|^{2} \\
& \leq c e^{-c_{3} h_{X}^{-\alpha}}|Y|
\end{aligned}
$$

with $c_{3}:=c_{2}-c_{1}>0$. Taking the same sequence of sets of centers $X_{n}$ as in Theorem 6.1 we see that the cardinality of $Y$ is only polynomial in $h_{Y}$, which means that we can bound two succeeding interpolants by

$$
\left\|s_{f, X_{k+1}}-s_{f, X_{k}}\right\|_{\Phi} \leq c e^{-\tilde{c}_{3} 2^{(k \alpha) / 2}} .
$$

This ensures the convergence of the telescoping sum.

\section{Saturation FOR Thin Plate SPLine interpolation}

In this section we concentrate on interpolation by thin plate or polyharmonic splines. To be more precise we consider the functions $\Phi_{d, \ell}=\phi_{d, \ell}\left(\|\cdot\|_{2}\right)$ with

$$
\phi_{d, \ell}(r)= \begin{cases}c_{d, \ell} r^{2 \ell-d} & \text { for odd } d \\ e_{d, \ell} r^{2 \ell-d} \log r & \text { for even } d\end{cases}
$$


with $d \leq 2 \ell$, where the constants

$$
\begin{aligned}
c_{d, \ell} & =\frac{(-1)^{\ell} \Gamma\left(\frac{d}{2}-\ell\right)}{2^{2 \ell} \pi^{d / 2} \Gamma(\ell)} \\
e_{d, \ell} & =\frac{(-1)^{(d-2) / 2}}{2^{2 \ell-1} \pi^{d / 2}(\ell-1) !\left(\ell-\frac{d}{2}\right) !}
\end{aligned}
$$

are determined by the fact that these functions should be the fundamental solutions of the iterated Laplacian (see Lemma 7.2).

The functions $\Phi_{d, \ell}$ are conditionally positive definite of order $m$ with $m=\ell-$ $\lceil d / 2\rceil+1$ on $\mathbb{R}^{d}$ and possess a generalized Fourier transform $\widehat{\Phi}_{d, \ell}$ which is $\|\cdot\|_{2}^{-2 \ell}$ up to a constant factor. Thus interpolants come from the space

$$
S(X)=\operatorname{span}\left\{\Phi_{d, \ell}(\cdot-x): x \in X\right\}+\mathcal{P}_{m}^{d}
$$

and Theorem 3.2 leads to the error bound

$$
\left\|f-s_{f, X}\right\|_{L_{\infty}(\Omega)} \leq c h_{X}^{\ell-\frac{d}{2}}\|f\|_{\Phi_{d, \ell}} .
$$

For a restricted set of functions $f$, an improvement in [12 yields

$$
\left\|f-s_{f, X}\right\|_{L_{\infty}(\Omega)} \leq c_{f} h_{X}^{2 \ell-d} .
$$

In [1] the following improved error estimate is given:

Theorem 7.1. Suppose $\Omega$ is a cube in $\mathbb{R}^{d}$ and the set of centers $X_{h}$ are given by the grid points $h \mathbb{Z}^{d} \cap \Omega$. If $f \in \operatorname{Lip}(2 \ell+1, \Omega)$, then the error can be bounded by

$$
\left\|f-s_{f, X_{h}}\right\|_{L_{\infty}(K)} \leq c_{f} h^{2 \ell}
$$

for every compact subset $K$ of the interior of $\Omega$ as $h \rightarrow 0$.

See [1] for the exact definition of the space $\operatorname{Lip}(2 \ell+1, \Omega)$. Note that this estimate is based on three additional assumptions:

- The function $f$ is supposed to be smoother than $f \in \mathcal{G}_{\Phi, \Omega}$. This is a natural assumption.

- The domain has to be a cube and the centers have to form a grid. This is a consequence of the proof given in [1]. A generalization to arbitrary centers would be useful.

- The estimates are restricted to compact subsets of the interior of the cube. This means that boundary effects are neglected.

Nonetheless, the result gives a hint about the possible local convergence order and we shall show that this order is also the saturation order. But before we can do that, we need two auxiliary results:

Lemma 7.2. For every test function $\gamma \in C_{0}^{\infty}\left(\mathbb{R}^{d}\right)$ and every $y \in \mathbb{R}^{d}$ we have

$$
\int_{\mathbb{R}^{d}} \Phi_{d, \ell}(x-y) \Delta^{\ell} \gamma(x) d x=\gamma(y) .
$$

A proof can be found in [6]. 
Lemma 7.3. Suppose $X=\left\{x_{1}, \ldots, x_{N}\right\} \subseteq \Omega$ is given. Suppose further that $\gamma \in$ $C_{0}^{\infty}(\Omega)$ satisfies $X \cap \operatorname{supp} \gamma=\emptyset$. Then for every $s \in S(X)$

$$
\left(\Delta^{\ell} s, \gamma\right)_{L_{2}(\Omega)}=0 \text {. }
$$

Proof. Choose an arbitrary $s(x)=\sum_{j=1}^{N} \alpha_{j} \Phi_{d, \ell}\left(x-x_{j}\right)+p(x) \in S(X)$. As $\Delta^{\ell} \mathcal{P}_{m}^{d} \equiv$ 0 we can use Lemma 7.2 to obtain

$$
\begin{aligned}
\left(\Delta^{\ell} s, \gamma\right)_{L_{2}(\Omega)} & =\sum_{j=1}^{N} \alpha_{j} \int_{\mathbb{R}^{d}} \Delta^{\ell} \gamma(x) \Phi_{d, \ell}\left(x-x_{j}\right) d x \\
& =\sum_{j=1}^{N} \alpha_{j} \gamma\left(x_{j}\right) \\
& =0 .
\end{aligned}
$$

Now we can give our saturation result.

Theorem 7.4. Let $\phi_{d, \ell}$ be any of the thin plate splines defined in (7.1). Suppose $\Omega \subseteq \mathbb{R}^{d}$ to be open and bounded, satisfying an interior cone condition. Suppose that for $f \in C^{2 \ell}(\Omega)$ the interpolating functions $s_{f, X}$ on $X$ satisfy

$$
\left\|f-s_{f, X}\right\|_{L_{\infty}(K)}=o\left(h_{X}^{2 \ell}\right) \quad \text { as } h_{X} \rightarrow 0
$$

for every compact subset $K$ of $\Omega$ and all $X \subseteq \Omega$. Then $f$ satisfies

$$
\Delta^{\ell} f=0 \quad \text { on } \Omega \text {. }
$$

Proof. Fix $x_{0} \in \Omega$. Choose $X \subseteq \Omega$ to be quasi-uniform with respect to a fixed $\delta>0$, such that $\min _{x \in X}\left\|x-x_{0}\right\|_{2}=c_{0} h_{X}$ with $c_{0}$ independent of $h_{X}$. Choose a test function $\gamma_{0} \in C_{0}^{\infty}\left(\mathbb{R}^{d}\right)$ with $\operatorname{supp} \gamma_{0}=B_{1}(0)=\left\{x \in \mathbb{R}^{d}:\|x\|_{2} \leq 1\right\}$ and $\int \gamma_{0}(x) d x=1$. Set $\tilde{h}=c_{0} h_{X} / 2$ and $\gamma_{h}:=\tilde{h}^{-d} \gamma_{0}\left(\left(\cdot-x_{0}\right) / \tilde{h}\right)$. Then the support of $\gamma_{h}$ is given by $B_{\tilde{h}}\left(x_{0}\right)$ and satisfies $B_{\tilde{h}}\left(x_{0}\right) \cap X=\emptyset$. Thus we can use Lemma 7.3 to get

$$
\begin{aligned}
\left(\Delta^{\ell} f, \gamma_{h}\right)_{L_{2}(\Omega)} & =\left(\Delta^{\ell}(f-s), \gamma_{h}\right)_{L_{2}(\Omega)} \\
& =\left(f-s, \Delta^{\ell} \gamma_{h}\right)_{L_{2}(\Omega)} \\
& \leq\|f-s\|_{L_{2}\left(B_{\tilde{h}}\left(x_{0}\right)\right)}\left\|\Delta^{\ell} \gamma_{h}\right\|_{L_{2}\left(B_{\tilde{h}}\left(x_{0}\right)\right)} \\
& \leq c \tilde{h}^{\frac{d}{2}}\|f-s\|_{L_{\infty}\left(B_{\tilde{h}}\left(x_{0}\right)\right)}\left\|\Delta^{\ell} \gamma_{h}\right\|_{L_{2}\left(B_{\tilde{h}}\left(x_{0}\right)\right)}
\end{aligned}
$$

with $s=s_{f, X}$. And because of

$$
\begin{aligned}
\left\|\Delta^{\ell} \gamma_{h}\right\|_{L_{2}\left(B_{\tilde{h}}\left(x_{0}\right)\right)}^{2} & =\tilde{h}^{-2 d} \int_{\mathbb{R}^{d}}\left|\Delta^{\ell} \gamma_{h}(x)\right|^{2} d x \\
& =\tilde{h}^{-d-4 \ell} \int_{\mathbb{R}^{d}}\left|\Delta^{\ell} \gamma_{0}(x)\right|^{2} d x \\
& =: \quad \tilde{h}^{-d-4 \ell} c_{0}^{2}
\end{aligned}
$$

we can conclude

$$
\left|\left(\Delta^{\ell} f, \gamma_{h}\right)_{L_{2}(\Omega)}\right| \leq c \tilde{h}^{-2 \ell}\|f-s\|_{L_{\infty}\left(B_{\tilde{h}}\left(x_{0}\right)\right)} .
$$

On account of the assumptions this leads to

$$
\lim _{h \rightarrow 0+}\left(\Delta^{\ell} f, \gamma_{h}\right)_{L_{2}(\Omega)}=0 .
$$


On the other hand we have

$$
\lim _{h \rightarrow 0+}\left(\Delta^{\ell} f, \gamma_{h}\right)_{L_{2}(\Omega)}=\lim _{h \rightarrow 0+} \int_{\mathbb{R}^{d}}\left(\Delta^{\ell} f\right)\left(x_{0}+h x\right) \gamma_{0}(x) d x=\Delta^{\ell} f\left(x_{0}\right)
$$

which proves $\Delta^{\ell} f\left(x_{0}\right)=0$.

Note that our proof also applies to the situation of classical splines. As in the latter case, functions in the saturation class are already determined by their values on the boundary of the domain:

Corollary 7.5. Suppose in addition to the assumptions of the last theorem that $\Omega$ has a $C^{\ell}$ boundary $\partial \Omega$. Then $f$ is already determined by the values of $\partial^{j} f / \partial^{j} \nu$, $0 \leq j \leq \ell-1$, on the boundary $\partial \Omega$. Here $\nu$ denotes the outer unit normal vector.

This sheds some light on the influence of boundary conditions on the possibilities to improve the approximation order $\ell-d / 2$ of (7.2) towards $2 \ell$.

Finally, we want to draw the reader's attention to the $d / 2$-gap arising not only in the discussion around (6.3), but also in (7.3) when compared to (7.4). If (7.2) could be improved by $h^{d / 2}$, then (7.3) would by [12] improve to $h^{2 \ell}$ and coincide with (7.4). We consider closing the $d / 2$-gap to be a challenging research task.

\section{REFERENCES}

1. Bejancu, A., Local accuracy for radial basis function interpolation on finite uniform grids, J. of Approx. Theory 99 (1999) 242-257. MR 2000e:41002

2. DeVore, R. A. and Lorentz, G. G., Constructive Approximation, Springer, Berlin, 1993. MR 95f:41001

3. Duchon, J., Sur l'erreur d'interpolation des fonctions de plusiers variables par les $D^{m}$-splines, R.A.I.R.O. Analyse numérique 12 (1978), 325-334. MR 80j:41052

4. Golitschek, M. von, and W. Light, Interpolation by polynomials and radial basis functions on spheres, Constructive Approximation 17 (2000), 1-18. CMP 2001:04

5. Jetter, K., J. Stoeckler, and J.D. Ward, Error estimates for scattered data interpolation on spheres, Math. Comp. 68 (1999), 733-747. MR 99i:41032

6. John, F., Plane Waves and Spherical Means Applied to Partial Differential Equations, Interscience Publishers, Inc., New York, 1955. MR 17:746d

7. Light, W. and Wayne, H., On power functions and error estimates for radial basis function interpolation, J. of Approx. Theory 92 (1998), 245-266. MR 98m:41006

8. Madych, W. R. and Nelson, S. A., Multivariate interpolation and conditionally positive definite functions, Approx. Theory and its Appl. 4 (1988), 77-89 MR 90e:41006

9. Madych, W. R. and Nelson, S. A., Multivariate interpolation and conditionally positive definite functions II, Math. Comp. 54 (1990), 211-230. MR 90e:41007

10. Schumaker, L. L., Spline Functions: Basic Theory, John Wiley \& Sons, New York,1981. MR 82j:41001

11. Schaback, R., Error estimates and condition numbers for radial basis function interpolation, Advances in Comp. Math. 3 (1995), 251-264. MR 96a:41004

12. Schaback, R., Improved error bounds for radial basis function interpolation, Math. Comp. 68 (1999), 201-216. MR 99d:41037

13. Schaback, R., Native spaces for radial basis functions I, in: M.W. Müller, M.D. Buhmann, D.H. Mache, and M.Felten (eds.), New Developments in Approximation Theory, ISNM Vol. 132, 255-282, Birkhäuser, Basel, 1999. CMP 2000:05

14. Schaback, R., A unified theory of radial basis functions (Native Hilbert spaces for radial basis functions II), J. of Computational and Applied Mathematics 121 (2000) 165-177. CMP 2001:01

15. Wendland, H., Sobolev-type error estimates for interpolation by radial basis functions, in: A. LeMéhauté, C. Rabut, and L.L Schumaker (eds.), Surface Fitting and Multiresolution Methods, 337-344, Vanderbilt University Press, Nashville, TN, 1997. MR 99j:65012 
16. Wendland, H., Error estimates for interpolation by compactly supported radial basis functions of minimal degree, J. Approx. Theory 93 (1998), 258-272. MR 99g:65015

17. Wu, Z. and Schaback, R. Local error estimates for radial basis function interpolation of scattered data, IMA J. of Numerical Analysis 13 (1993), 13-27. MR 93m:65012

Institut Für Numerische Und Angewandte Mathematik, Universität Göttingen, Lotzestr. 16-18, 37083 Göttingen, Germany

E-mail address: schaback@math.uni-goettingen.de

Institut für Numerische und Angewandte Mathematik, Universität Göttingen, Lotzestr. 16-18, 37083 Göttingen, Germany

E-mail address: wendland@math.uni-goettingen.de 